\author{
D. E. Zakrzewska ${ }^{1,2}$, A. K. Krella ${ }^{*}$ \\ ${ }^{1}$ Institute of Fluid Flow Machinery, Polish Academy of Sciences, Fiszera 14, 80-231 Gdansk, \\ Poland \\ ${ }^{2}$ Gdańsk University of Technology, Faculty of Mechanical Engineering, 11/12 Narutowicza, \\ 80-233 Gdansk, Poland, \\ *akr@imp.gda.pl
}

\title{
CAVITATION EROSION RESISTANCE INFLUENCE OF MATERIAL PROPERTIES
}

\begin{abstract}
The cavitation erosion is the phenomena that causes degradation of fluid flow machinery components due to repetitive implosion of cavitation bubbles adjacent to the solid surface. Cavitation erosion is a complex phenomenon, which includes not only hydrodynamic factors of liquid, but also properties of erodible material e.g. microstructure, hardness or Young modulus. In order to reduce the negative impact of erosion on machine components, there are many methods to increase cavitation erosion resistance. The paper discusses the correlations between structural and mechanical properties and the resistance to cavitation erosion (CER) of pure materials, their alloys and coatings. Methods to increase CER have also been described - using heat / thermochemical treatment and application of coatings by various methods.
\end{abstract}

Keywords: cavitation erosion, erosion, resistance, material properties

\section{INTRODUCTION}

According to the ASTM G32 standard, cavitation is defined as the formation and subsequent collapse, within a liquid, of cavities or bubbles that contain vapor, gas or a mixture of vapor and gas [ASTM G-32-10 Standard]. Bubbles filled with vapor or dissolved gases form in low pressure regions and implode violently in areas of higher pressure. During bubbles implosion the micro-jets and the shock waves are generated, which are called cavitation pulses due to their short duration. The repeated impacts of the micro-jets and the shock waves can cause e.g. mass loss of material, this phenomenon is known as cavitation erosion. This affects the life of the devices [1].

Cavitation erosion depends on the physical and chemical properties of liquid and material, and also on the development of cavitation called as cavitation intensity, which can be expressed by the equation [2]: 


$$
I=\frac{K}{T} \frac{1}{2 \rho c} \sum_{k=1}^{M} n_{k} p_{k}^{2}
$$

Where $K=10^{-5}$ (proportional coefficient), $T$ total measurement time, $\rho$ - density of the liquid, $c$ - sound velocity, $M$ - number of pressure intervals, $k$ - the consecutive number of the interval, $n_{k}$ - number of pulses in the interval, $p_{k}$ - value of pressure amplitudes in k-th interval.

The resistance of material to cavitation erosion is presented by the curves of mass loss in exposure time. Instead of mass loss, volume loss or erosion rate in exposure time can be shown (Fig.1) (according ASTM G32-10).

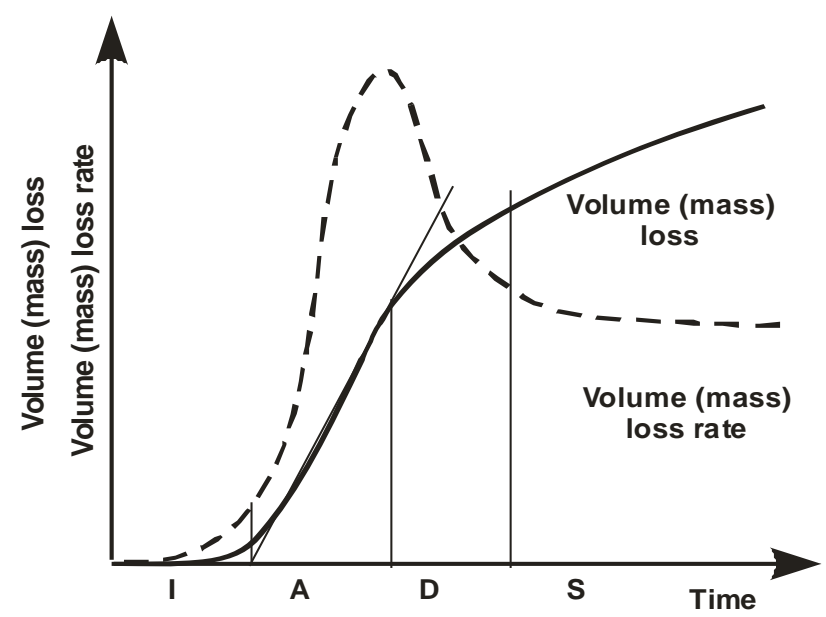

Fig.1. Cavitation curve with periods of degradation

The cavitation curve allows detecting following periods of material degradation: incubation (I), acceleration (A), deceleration (D) and steady state (S). During incubation period, material accumulates impact energy, undergoes elastic and plastic deformation, and the properties of surface layer are changed. The acceleration period is characterized by intensive material loss. At the end of this period erosion rate achieves its maximum value. Cavitation erosion extends over the entire area subjected to cavitation. Changes of surface geometry cause the start of the next period, which is the deceleration period and is characterized by progressive decrease of erosion rate. The impacts of cavitation pulses are amortized by a liquid filling the pits. As a result less impact energy is delivered to material surface layer. The last period of degradation is steady state period, during which the erosion rate is almost constant due to a slight changes in surface geometry structure [3].

The duration of the mentioned erosion periods depends on the material properties, the type of cavitation, cavitation number and cavitation intensity.

Cavitation damage is a serious problem not only in hydro turbines [4], but also in other hydraulic devices, e.g. propellers (the interaction between a ship's propeller and the nonuniform wake behind the ship) or pump impellers. Rayleigh was the first person who reported this type of damage on ship propellers. Degradation is mainly caused by the coherent collapse of a cloud of cavitation bubbles, which is more violent than that of individual bubbles and accompanied by intense noise [5], [6].

Although the cavitation phenomenon is very undesirable due to the risk of the occurrence of erosion, in some circumstances, some effects of cavitation are applied in advanced 
oxidation of substances that pollute the natural environment [7], e.g. in decomposition of selected organic compounds, which hardly undergo biodegradation, intensification of biological wastewater treatment, ultrasonic emulsification and synthesis of biodiesel [8].

Due to harmful effects of cavitation, there are some ways to their prevention. Karimi and Martin [9] classified these methods into three groups. The first group includes improvement of the hydrodynamic designs, the second group includes the design of high cavitation erosion resistance materials and the last group includes simultaneous changes in material properties and hydrodynamic design to diminish cavitation intensity.

In order to predict the erosion rate, it is important to determine the relationship between the material properties and their cavitation resistance. However, the correlations found so far are not universal and usually cover a limited group of materials [10]. The aim of this paper is to present properties of eroded material affecting the cavitation erosion and their correlation with cavitation erosion resistance, and possible ways to increase resistance to cavitation erosion.

\section{CAVITATION EROSION OF PURE METALS AND METAL ALLOY STRUCTURES}

The cavitation erosion resistance depends on material properties (mechanical, endurance and structural), liquid properties (e.g. temperature, chemical composition, chemical aggressiveness, viscosity) and cavitation intensity. According Fig. 2., the highest cavitation erosion resistance has material with homogeneous and fine-grained structure, high endurance and mechanical properties, and high corrosion resistance. To the key material properties belongs hardness, tensile strength, yield strength, strain energy, fracture toughness and fatigue strength. Therefore, their effect on erosion resistance is described below.

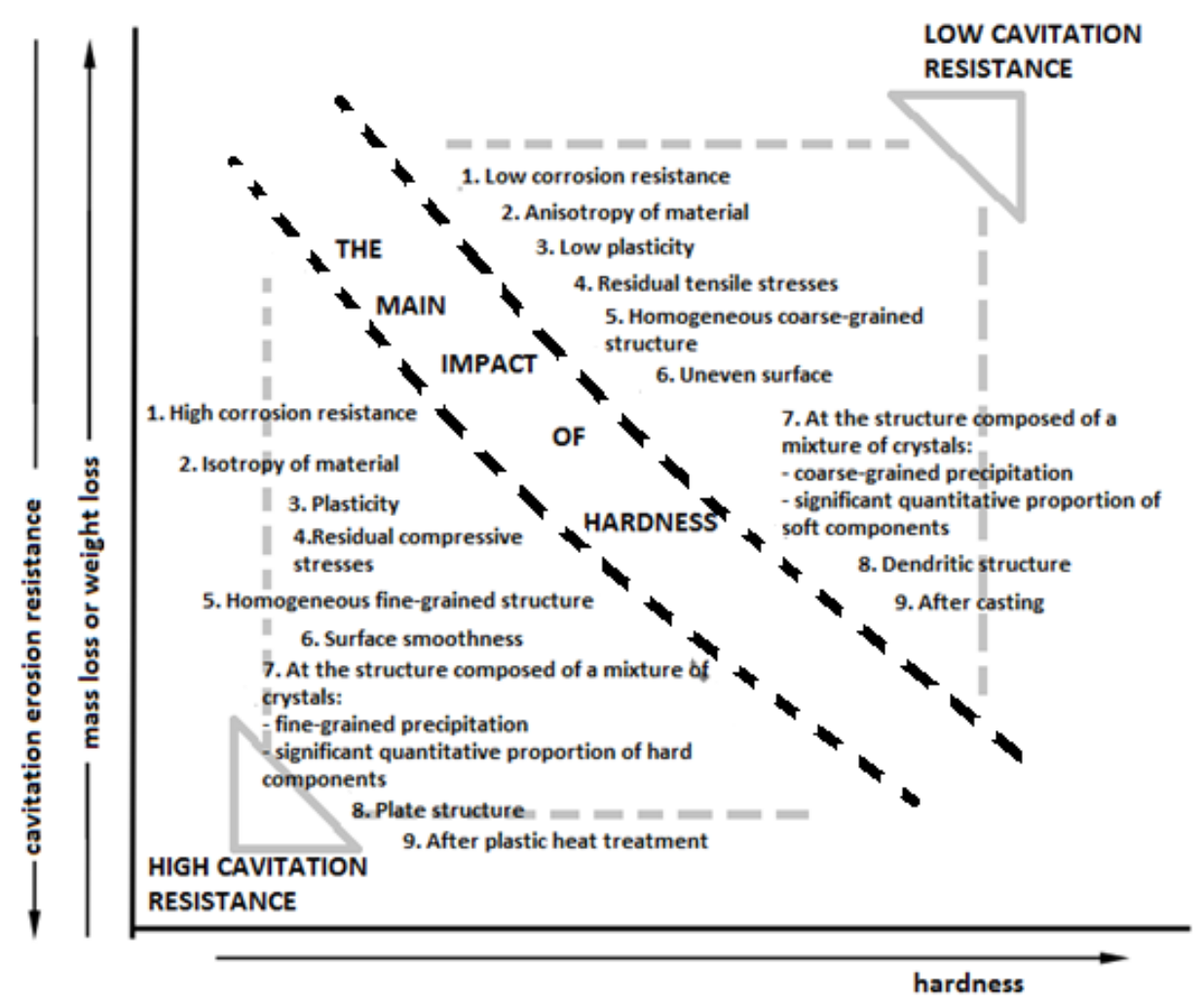

Fig. 2. Relation between cavitation erosion resistance mass or weight loss as function of hardness for different material properties [11] 


\section{HARDNESS}

Hardness is the most often mentioned property, which has an influence on the erosion resistance [1][4]. Hardness is described as the resistance of material to withstand indentation. This property is related with the other material properties e.g. Young's modulus, and also plasticity and strength. Heymann noted the existence of an exponential correlation between hardness ( $\mathrm{HV}$ ) and the normalized resistance to cavitation erosion $\mathrm{N}_{\mathrm{e}}$ defined as the ratio of the tested material's resistance to the resistance of the reference material tested at similar test parameters in a following form [3]:

$$
N_{e} \sim H V^{n}
$$

Eq. 1 works for various groups of materials: stainless steels, cast steel, bronzes, brass and aluminum alloys. In addition, Heymann [3] obtained that the normalized erosion resistance $(\mathrm{Ne})$ increases with the hardness of the material in exponent of $n=\frac{5}{2}$. His correlation between hardness and cavitation erosion resistance (Eq. 1) has been used in many works [9], [12][14].

Richman and McNaugthon [15] analyzed results of Knapp's investigation also paying attention that the best correlation is the logarithmic correlation between hardness and MDP (mean depth penetration) with the correlation coefficient equals 0.813 .

Investigations of four Fe-Mn-Al alloys and 304 stainless steel [16] in distilled water confirmed an increase the cavitation erosion resistance with material's hardness (Fig. 3).

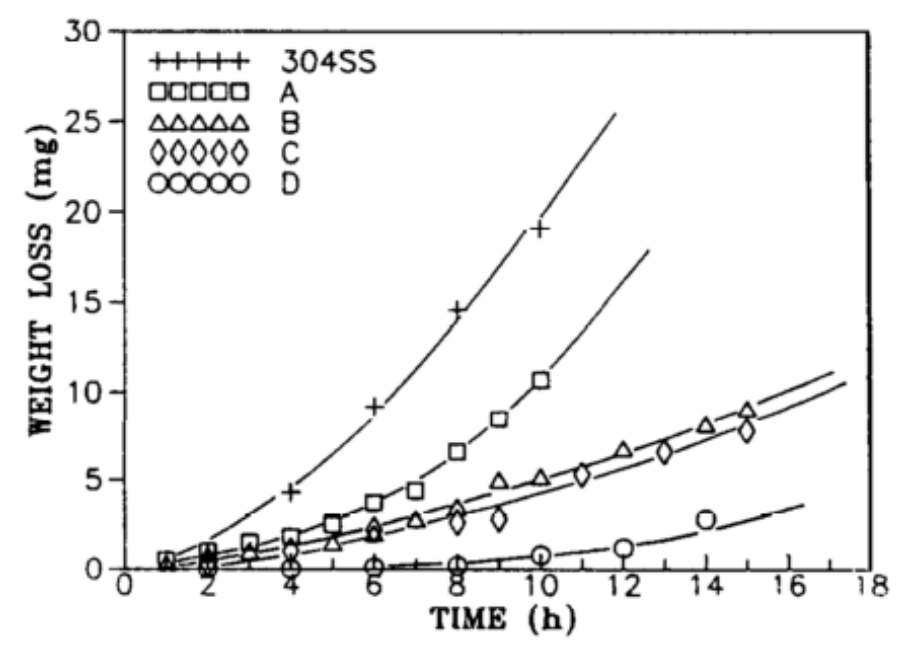

Fig. 3. The cumulative weight loss of different Fe-Mn-Al alloys (in order of decreasing hardness and strength: C, D, B, A) after heat treatment and 304 stainless steel in distilled water [16]

However, tests performed in $3.5 \% \mathrm{NaCl}$ solution showed that besides hardness also ability to passivation is beneficial for the cavitation erosion resistance. Kwok et al. [17] investigated an effect of heat treatment of $\alpha+\beta$ brass and S30400 steel on the materials' properties and the cavitation erosion resistance. They obtained an exponential correlation between the cavitation erosion resistance $(C E R)$ and following property: hardness, tensile strength, engineering strain energy and even grain size for both materials. They found that the correlations have the following form [17]: 


$$
C E R=k(X)^{a},
$$

where $k$ and $a$ are constants different for each material property, including grain size, $X$ is hardness, tensile strength and engineering strain energy.

Similar correlation of $C E R$ with hardness have been found by Tzanakis et al. [18]. According to Ref. [18], the normalized cavitation erosion resistance calculated as a ration of the cavitation erosion resistance $(C E R)$ to the maximum cavitation erosion resistance $\left(C E R_{\max }\right)$ can be expressed in a following form:

$$
C E R / C E R_{\max }=a(H)^{n}
$$

with a determination coefficient $\mathrm{R}^{2}=0.79$.

Hattori et al. [19] basing on experimental data of 15 types carbon steels tested at various test methods have found a direct impact of hardness on the cavitation erosion resistance. The correlation is described by equation:

$$
\left(N_{e}\right)_{X}=2,1 \operatorname{Exp}(-6) \times H V^{2,4}
$$

where $N_{e}$ is normalized erosion resistance of arbitrary material X.

Statistical analysis of a database of 13 kinds of stainless steels with various structure (austenite, martensite, ferrite and duplex) tested at vibratory devices allows Hattori and Ishikura [20] to correlate $C E R$ with hardness by equation:

$$
C E R=2,6 \operatorname{Exp}(-7) \times\left(H V \times F_{\text {mat }}\right)^{2,4}
$$

Where $F_{m a t}$ is material factor equals a ratio of hardness of eroded surface to that of original surface.

Despite many studies showing the positive effect of an increase in material hardness on an increase in cavitation resistance, a comparison of a wide range of materials, including austenitic steels, carbon steels, intermetallic alloys and cemented carbides, does not confirm the existence of such universal correlation [9][20][21]. The mentioned correlation is observed only within individual groups of materials, e.g. low-carbon steels, aluminium alloys and titanium alloys [22].

\section{TENSILE STRENGTH}

Besides hardness, also tensile strength $\left(\sigma_{\mathrm{y}}\right)$ and ultimate tensile stress $\left(\sigma_{u}\right)$ are used to correlate with the cavitation erosion resistance. Investigations of the cavitation erosion resistance of copper and nickel alloys allow to propose the correlation between ultimate tensile strength and Maximal Depth Penetration Rate (MDPR) for by equation [23]:

$$
(M D P R)^{-1}=k\left(\sigma_{u}\right)^{2,3}
$$

where $k$ is constant.

Garcia and Hammitt [24] offered the correlation for several groups of materials (aluminum alloys, stainless steels, cooper alloys, nickel alloys) describing MDPR by equation: 


$$
M D P R=-1,570+6,48 \times 10^{2}(\sigma)^{-1 / 2}
$$

Hattori and Ishikura [20] confirmed an impact of tensile strength on the cavitation erosion resistance of steels (Fig. 4). The increase of the cavitation resistance together with the increase of tensile strength was also confirmed in Refs [14], [25]-[27]. For this reason, methods that improve ultimate tensile strength are considered beneficial for improving CER. Friction stir processed improves hardness and also ultimate tensile strength, which increase CER [28]. However, as emphasized in Ref. [27], tensile strength not alone, but together with other material properties, including fatigue strength, stacked energy consumption (SFE) determine $C E R$.

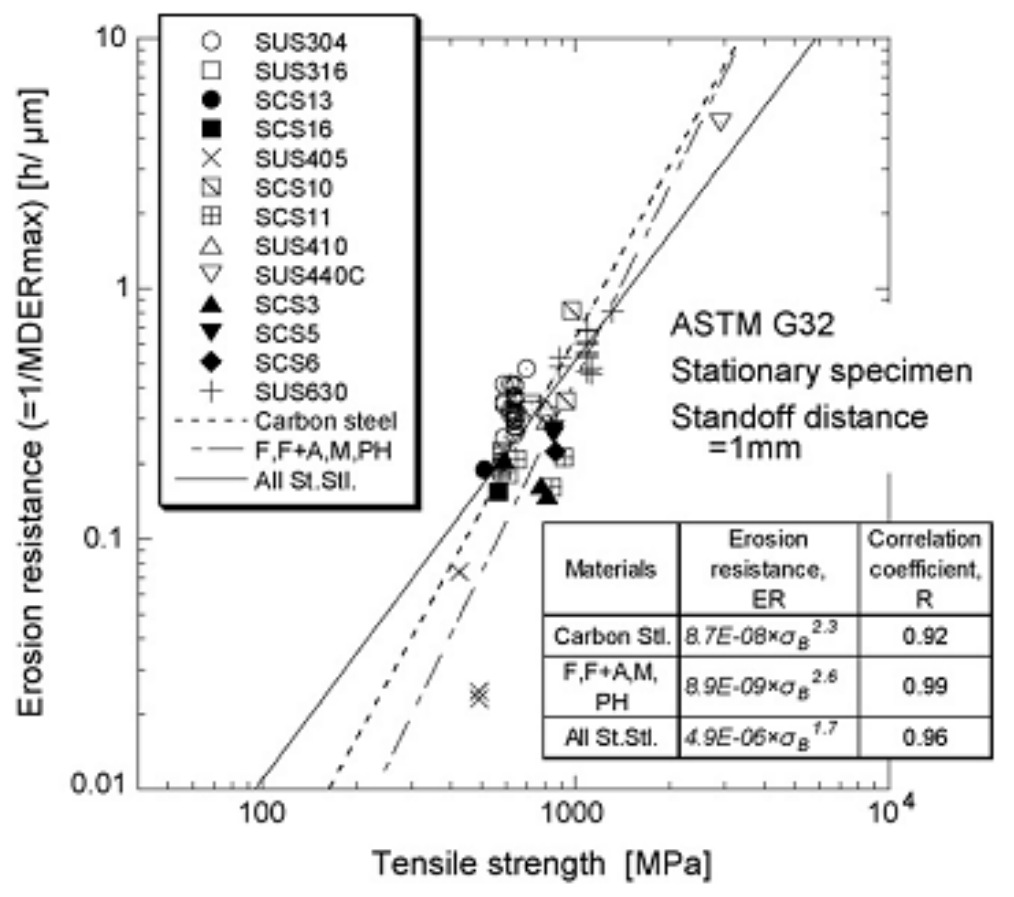

Fig. 4. Relation between tensile strength and erosion resistance for steels [20]

Investigation of Cr-Co-Ni-Mn austenitic stainless steels [14] proved that an increase of tensile strength as well as yield strength increase the incubation period and CER. In Ref. [29] was shown that high yield strength connected with high toughness and ductility improve $C E R$ of high-nitrogen austenitic stainless steel (HNAS) in comparison to two CrNiMo stainless steels even 3.3 times.

On the other hand, Sreedhar et al [30] described inversely proportional influence of yield strength to $C E R$ for commercial aluminum alloys.

High yield strength affects the longer incubation time and better cavitation erosion resistance in steel Cr-Co-Ni-Mn compared to 304 stainless steel. High yield strength also has a beneficial effect on $C E R$ of titanium alloy and TiNiNb alloy [14]. Bregliozi et al. [25] noticed that an increase of yield strength as well as hardness and tensile strength influence the increase of CER of 304 austenitic stainless steel. Feller and Kharrazi [12] comparing an effect of tensile strength on incubation time for pure metals and their alloys, noticed that yield strength of material is not the dominant factor to describe its $C E R$. Similar relationship was obtained by Thiruvengadam [13] who investigated different group of materials. However, 
Richman and McNaughton [15] noticed that it is possible to correlate yield strength with $C E R$, but it is important to remember that yield strength is roughly correlated with incubation time (Fig. 5).

The influence of yield strength on CER is confirmed also in Ref [31] (Fig. 6). On the other side, high entropy alloys [32] with low yield strength have better CER than $316 \mathrm{~L}$ stainless steel.

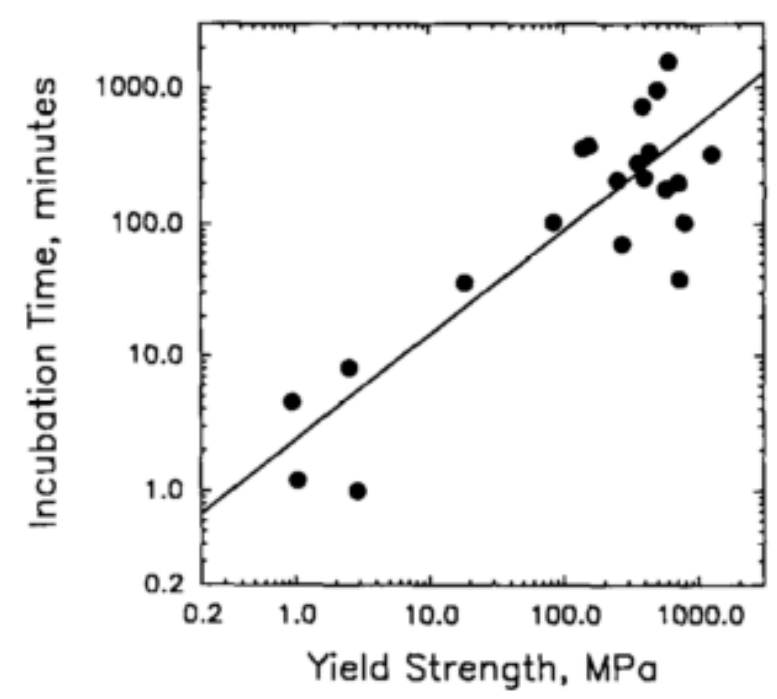

Fig. 5. Yield strength and incubation time correlation in vibratory apparatus in Feller and Kharrazi investigation [15]

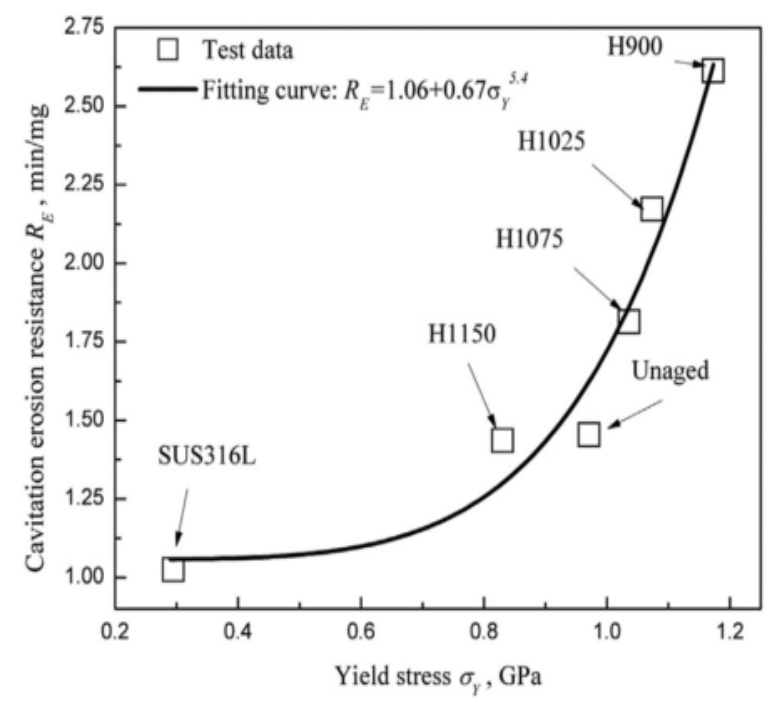

Fig. 6.Correlation between cavitation erosion resistance and yield stress [25]

\section{STRAIN ENERGY}

Strain energy $(U)$ is the energy stored in the material due to deformation caused by external forces. The accumulated energy caused by plastic deformation is called plastic strain energy or in case of elastic deformation - elastic strain energy [23].

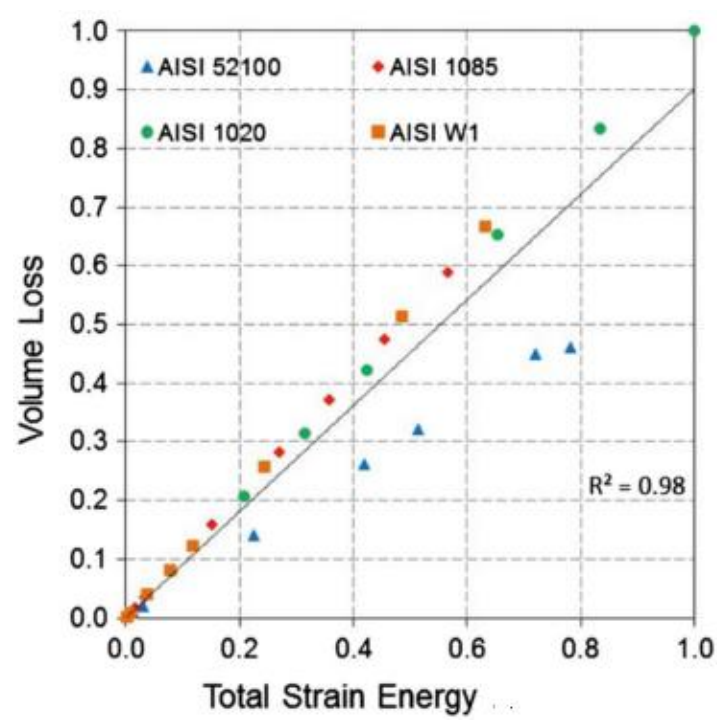

Fig. 7. Relationship between total strain energy and volume loss for steel used in Ref. [18] 
Thiruvengadam and Waring [13] proved that strain energy plays a significant role in $C E R$. Comparing estimated strain energy $\left(U_{e}\right)$ and true strain energy $\left(U_{e}^{*}\right)$ of various metals on the resistance to cavitation erosion, the linear relationship between volume loss rate and strain energy was found [13]. This relationship was better than for other mechanical properties.

Mann [33] reported that boronizing $13 \mathrm{Cr}-4 \mathrm{Ni}$ steel resulted high hardness and low elongations, and finally low $C E R$ in comparison to in as-received state or boronizing and tempering state of this steel. The best $C E R$ had this steel in as-received state due to the highest strain-energy. Similarly, excellent correlation of $C E R$ of three commercial steel grades (AISI 52100, AISI 1020, AISI 1085) with their strain energy has been found in Ref. [18] (Fig. 7). However, in Ref. [34] has been pointed that CER is better described by a combination of several properties, e.g. of ultimate tensile strength and elastic modulus or ultimate resilience and hardness, absorption energy and fatigue strength than the only one property.

\section{FATIGUE STRENGTH}

Besides hardness, a property that has a dominant effect on $C E R$ is fatigue strength $\sigma_{f}$ [15], [35]. This was affirmed by Richman and McNaughton [15] comparing and analyzing the results obtained by Feller and Kharrazi [12] and Knapp [36]. They noticed that fatigue strength is responsible for the material's resistance to the cavitation erosion phenomenon in $96 \%$. The exponential relationship of fatigue strength and mean depth penetration (a) as well as the relationship of fatigue strength and maximum erosion rate (b) is shown in Fig. 8.
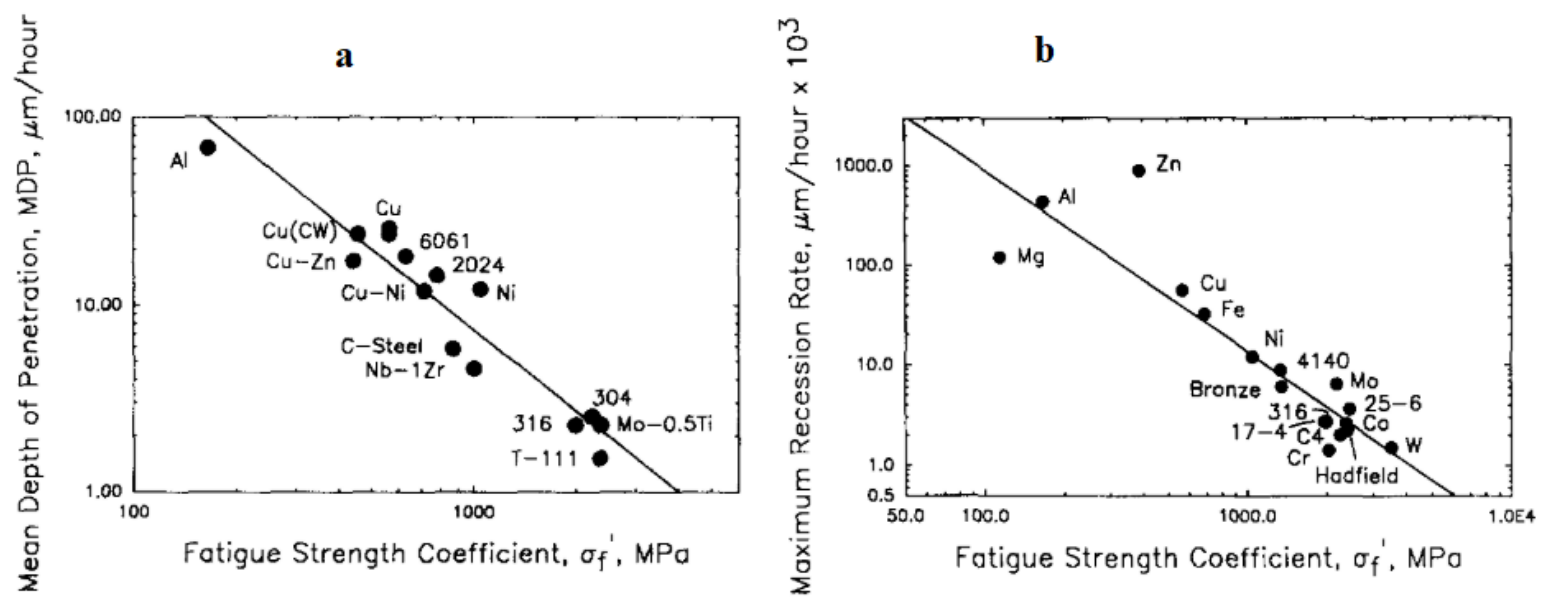

Fig. 8. Correlation between fatigue strength coefficient and mean depth penetration (a), maximum recession rate (b) [15]

Also in Ref. [37], there was confirmed significant impact of fatigue strength to CER and showed beneficent effect of explosive cladding of NiTi, which have high fatigue strength coefficient combined with a high cyclic strain-hardening exponent, on CER.

Będkowski et al. [35] performing fatigue and cavitation erosion tests have proved a correlation between CER of the steels (10HNAP, 18G2A, 15G2ANb) and their fatigue strength determined under uniaxial random tension-compression by following equation:

$$
\log \sigma_{R M S}=1,534+0,425 \log p_{1}
$$

where: $\sigma_{\text {RMS }}$ - root-mean-square value of stress, $p_{1}$ - pressure in the nozzle inlet in cavitation jet made by Lichtarowicz. 
Chen et al. [38] proposed two parameters $\mathrm{T}$ and $\mathrm{N}$ for predicting CER based on an assessment that cavitation erosion is the result of strain fatigue failure. The parameter $\mathrm{T}$ (the uniform deformation strengthening modulus of material, $[\mathrm{MPa}]$ ) and the parameter $\mathrm{N}$ (considered as the impact load property, $[\mathrm{J} / \mathrm{m}])$ are described by the formulas [38]:

$$
\begin{gathered}
T=\frac{\sigma-\sigma_{0, z}}{s_{f}} \\
N=\frac{E_{i}}{S_{i}}+\frac{E_{p}}{S_{p}}
\end{gathered}
$$

where: $\varepsilon_{f}$ - true strain obtained in static tensile test, $\varepsilon_{f}=\ln (1-\Psi), \Psi$ - reduction of crosssectional area, $\sigma$ - tensile strength, $\sigma_{0,2}$ - yield strength, $E_{i}$ - crack formation work, $E$ - total energy (work) that the specimens absorbed during fracture, $S$ - the maximum offset at the point of fracture, $E_{p}$ - crack extension work, $S_{i}, S_{p}$ - are the respective offset corresponding to $E_{i}$ and $E_{p}$.

Based on the above mentioned parameters, the average weight loss rate of steel $\left(W_{c}[\mathrm{mg} / \mathrm{h}]\right)$ was proposed to calculate from a following formula [38]:

$$
W_{c}=\alpha \frac{V^{2}}{\sqrt{N T}}
$$

Where: $v$ is water velocity $[\mathrm{m} / \mathrm{s}], \alpha$ is a material constant.

Using the above formula, a comparative analysis with experimental data for four steel (16CrNi4Mo, Q235, M50NiL, 0Cr13Ni4Mo) was conducted (Fig. 8).
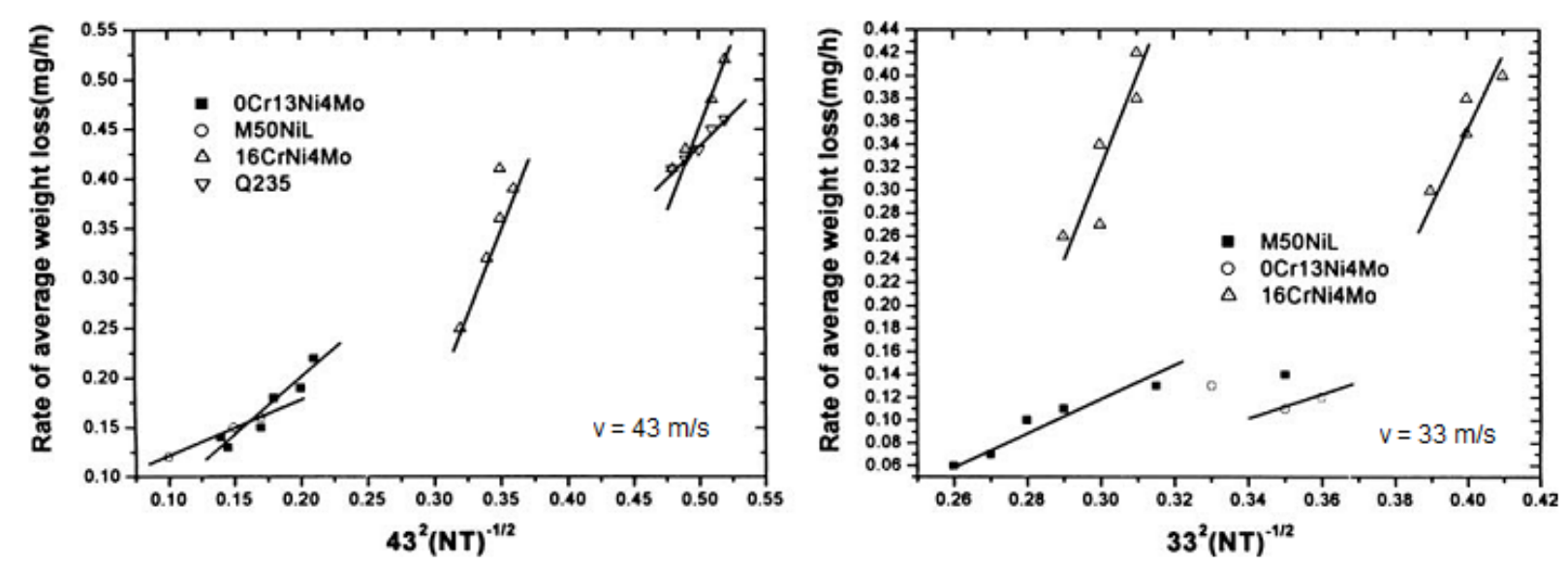

Fig. 8. Correlation of weight loss rate with Wc parameter [38]

The observed differences in Fig. 8. were associated with the $\alpha$ parameter, which is related to other mechanical properties. It was also noticed an increase of $C E R$ with a simultaneous decrease in the value of $\alpha$ parameter.

The fatigue strength determine also CER of plastics. Hattori and Takamoto [39] showed a linear relationship between $C E R$ and the impact energy and the fatigue strength. In addition, fatigue approach (the Palmgreen-Miner rule and the Paris- Erdogan law) have been also used for predicting mass or volume loss in cavitation erosion tests [40], [41]. 


\section{STRUCTURE}

In addition to mechanical properties, $C E R$ depends on the structure of the material. According to Ref. [25], CER is related to the grain size. AISI 304 stainless steel and HN steel exhibited an increase in mechanical properties (hardness, tensile strength, yield strength) along with the decreasing grain size. This contributed to increase CER. Furthermore, fine grain steel characterized better corrosion resistance compared with medium and coarse grain steel. In Ref. [42], it is showed that in standard polycrystalline metals $C E R$ increases with decreasing grain size. Better mechanical properties due to fine grain for steel also confirmed in Ref. [32], however, corse grain size in high entropy alloys or low nickel austenitic stainless steel [42] results in high ductility. Small grain sizes ensure good CER also for applying coatings [43], even 3 to 6 time better [44] than pure steel.

Another method to enhance CER of the steel AISI 316L was friction stir processing (FSP) [32]. Cavitation erosion tests made according to ASTM G-32 have shown that mass loss of 316L steel after FSP was significantly lower than for unprocessed steel. Moreover, the incubation time was even ten times longer after friction stir processing. Also, surface microhardness of the FSP samples was higher approximately two times than base material. This confirms that, grain size influences surface hardness and CER which is in accordance with previous mentioned impact of hardness on CER. Microscopic studies revealed that $316 \mathrm{~L}$ steel exposed to the lowest rotation speed during FSP process exhibits more homogenous microstructure after CE tests than 316L steel exposed to high rotation speed during FSP and base material (large and deep erosion craters). The common feature of all microstructures gained for $316 \mathrm{~L}$ steel exposed to cavitation is a surface morphology typical of ductile fracture. As highlighted in Ref. [32], fine-grained steel during cavitation impact indicates less damage than coarse-grained steel, as a result of higher work hardening rate due to micro-jet impingements.

Also it has been reported in the literature that usually, ferritic stainless steel has lower CER than austenitic and martensitic stainless steel [45]. The cavitation resistance of materials beyond microstructure is also influenced by e.g., roughness [46], composition and phase transformation [47].

The simplest method of improving CER is to increase content or to add component that affects the change of material properties. This method was applied in Ref. [48], in which is described a reinforcement $C E R$ of steel $316 \mathrm{~L}$ by adding molybdenum. Samples were made by mixing and melting mixture of pure $(99,9 \% \mathrm{wt})$ components desirable steel and hot rolling into plates. Using this method allowed to obtain samples with Mo content 2,5\%, 4\%,6\% and $8 \%$. Cavitation erosion tests proved that with increasing Mo content decreases mass loss and mean depth of erosion (MDE). Microstructure analysis of all samples after cavitation erosion tests shown that, with the higher molybdenum content, the roughness decreased. In addition, the surface of the sample with the highest Mo content showed slight damage. This confirms that $C E R$ can also be elevated by addition to steel molybdenum. Allenstein et al [49] improved about 5-6 times cavitation erosion resistance Ca-6NM martensitic stainless steel by low-temperature plasma nitride in comparison to non-nitrided steel Ca-6NM. They also noticed that longer nitriding time translates to higher cavitation erosion resistance.

\section{COATINGS}

Undoubted effect of hardness on CER contributed to searching for methods of increasing material hardness or surface hardness. The most popular is thermal or thermochemical treatment, especially nitriding [31], [49], [50], which increase hardness and also improves 
other mechanical properties. The other way for increasing surface hardness is deposition of hard coatings (e.g. WC-Co [51]) by means of various methods e.g. High Velocity Oxy-Fuel Thermal Spray (HVOF) [52], [53], PVD [54], [55], [56]), Vacuum Plasma Spraying (VPS) [57], Atmospheric Plasma Spraying (APS) [58], arc spraying (ARC) [44], or Plasma Assisted Physical Vapour Deposition (PAPVD) [59]. Friction stir process (FSP) [28] is, as mentioned, another surface treatment used to reduce grain size and improve CER. Application of the coating allows not only to obtain the appropriate surface hardness, but also other mechanical properties that may effect on $C E R$, e.g. high wear resistance, which can be beneficial in situations where surface wear or erosion can occur [51]. Kim and Lee [60] proved strongly dependence between hardness and CER of coatings produced by arc-spraying. However, as emphasized in Ref. [61], along with increasing hardness increases brittleness, which has an effect on decreasing CER. The active screen low temperature plasma nitriding increased both hardness and CER of martensitic stainless steel AISI 410 [10]. Mass loss was approx. 28 time less than this steel without mentioned treatment. Comparison two surface treatments PAPVD and plasma nitriding proved that a combination of plasma nitriding followed by deposition of $\mathrm{Cr}_{1-\mathrm{x}} \mathrm{N}_{\mathrm{x}}$ coating using PAPVD method is a better solution for increasing $C E R$ than just a single surface treatment [59].

As was mentioned, deposition of coatings is used to improve CER. HVOF coatings belong to coatings that increase CER [62]. Taillon et al. [63] used HVOF method to deposit $\mathrm{Fe}_{3} \mathrm{Al}$ and $\mathrm{Fe}_{3} \mathrm{Al}$ reinforced with nitride and boride phases coatings from pure powders. Their results were compared with coatings made from commercial powders e.g. WC-CoCr and $\mathrm{Cr}_{2} \mathrm{C}_{2}$-NiCr. As a substrate for HVOF coatings was used steel AISI 444. The researchers also investigated low carbon martensitic stainless steel, 13Cr-8Ni (S13800), 13Cr-4Ni (S41500), 15Cr-5Ni (S15500) and ferritic stainless steel AISI 444. All coatings exhibit lower erosion rates than bare steels, but no correlation of $C E R$ with hardness, young modulus or surface properties has been found. Microstructure analysis of eroded surfaces showed that cracks nucleation starts in the matrix for composite coatings and in grain boundaries of ferritic steel. The investigated coatings exhibit comparable erosion rates to commercial powders. Wu et al. [62] proved that WC-Co-Cr coating sprayed by HVOF method has 64\% lower mass loss compared to stainless steel $1 \mathrm{Cr} 18 \mathrm{Ni} 9 \mathrm{Ti}$.

The atmospheric plasma spraying (APS) [64] allows to create low porous and hard YSZNiCrBSi composite coatings, which also strengthen the surface of the material for cavitation erosion. Better $C E R$ is a result of denser composite microstructures, although cavitation damage can occur on YSZ-NiCrBSi splats interfaces, which may also influence on higher mass loss rates during cavitation exposure. The APS method also allows creating the CoMoCrSi coatings [34] in which we can observe hard precipitates (cobalt-molybdenumsilicon based intermetallic Laves phases) dispersed in a softer cobalt-based alloy matrix. This coating has also high cavitation erosion resistance, but it is higher after heat treatment and compared with HVOF coatings is lower.

High density and low porosity coating, with high adhesion to the substrate is also possible by arc spaying [46]. However, in the case of the applied coating, it was noticed that it was necessary to grind the surface, which affected the increasing CER. Stella et al [57] deposited NiTi coating using vacuum plasma spraying (VPS) on stainless steel 316L. For comparison was used pre-alloyed $\mathrm{Ni}-\mathrm{Ti}$ powder prepared by the manufacturer by gas atomization (c1), and powder of $\mathrm{Ni}$ powder and $\mathrm{Ti}$ powder blended in tubular mixer (c2). Cavitation erosion tests showed pronounced bigger mass loss for coating $\mathrm{c} 2$ than coating $\mathrm{c} 1$. Also bulk material did not exhibit any mass loss in 30 hours of tests, after this time (to the end of tests) bulk material exhibit less than $4 \mathrm{mg}$, while for the coating c2 total mass loss was 12.8 $\mathrm{mg}$ and for the coating $\mathrm{c} 1$ was $5.6 \mathrm{mg}$, which was explained by low adherence particles in the 
pores. Moreover, a high value of surface porosity was noted for tested coatings. EDX analysis revealed presence of additional $\mathrm{Ni}_{3} \mathrm{Ti}$ and $\mathrm{Ni}_{4} \mathrm{Ti}_{3}$ phases in c2 coating, and NiTi phase in c1 coating. Mean depth of erosion (MDER) had the lowest value for c2 coating. Analysis of coatings microstructure during the cavitation erosion tests were also performed. After 22 hours of cavitation tests, not all damages in c1 coating can correspond initial defects. According to Stella et al [57], the NiTi grains could be affected by stress generation or crack propagation. Thorough analysis of microstructure showed undeformed grains and intergranular cracks on grains boundaries in c1 coating. Microstructure of the coating c2 showed damage of the $\mathrm{Ni}_{3} \mathrm{Ti}$ phase mostly located along phase boundaries, what was explained of the accumulated stresses between two phases characterized by various deformations.

Comparison of the cavitation erosion resistance of $\mathrm{WC}-10 \mathrm{Co} 4 \mathrm{Cr}$ coatings, Co-based coatings, WC-10Co4Cr/Co-based composite coatings, and Fe-based amorphous / nanocrystalline coatings produced by HVOF method on $316 \mathrm{~L}$ steel in artificial sea water and deionized water showed the highest $C E R$ of WC-10Co4Cr coatings while maintaining high corrosion resistance [65]. In addition, the coating with the highest $C E R$ had the highest surface hardness (1273 HV), which confirms earlier considerations regarding the high association of surface hardness with $C E R$.

In Ref. [66], there was highlighted an influence of HVOF spraying parameters on composition, microstructure and mechanical properties of Co-based coatings, and their effects on $C E R$ of $316 \mathrm{~L}$ stainless steel substrate. It has been shown that the higher the flame temperature during coating deposition, the hardness and toughness of the coatings were improved and decreased the roughness of surface.

According to Ref. [67], deposition of $\mathrm{ZrC}$ nanoceramic coating by the double glow disarge sputter technique on steel 316 also improved CER and increased corrosion resistance in comparison to uncoated steel.

Another example of an effect of coatings deposition on CER is shown in Ref. [68], in which $8 \mathrm{wt} \%$ yttria-stabilized zirconia (8YSZ) and 8YSZ-ER coatings deposited on 316 steel were investigated. In addition, NiCrAlY was introduced into the micro defects of the assprayed 8YSZ coating by vacuum impregnation. The addition of NiCrAlY to the 8YSZ coating enabled to improved cohesion strength, toughness, and hardness of the coating. This increased the CER three times compared to the 8YSZ coating. According to [68], the coating absorbs the shock wave resulting from bubble collapse, using it for recrystallization, which increased CER.

The magnetron sputtered AlTiN and TiAlN coatings deposited on SS304 stainless steel [67] showed better AlTiN thin flim adhesion to the steel substrate than TiAlN thin film, which was correlated with surface hardness. The results obtained highlight the important impact of structural and mechanical properties (hardness, adhesion, and elastic modulus) on CER. With an increase in adhesion of the coating increased CER. CER of AlTiN coating was three times higher comparing with TiAlN coating, and ten times compared with uncoated steel. In addition, sliding wear of both coatings was 24 times lower than uncoated steel, and deposition of coating caused a decrease of the friction coefficient two times. Similar results of the role of adhesion of PVD coatings on CER were obtained in Refs [55], [56] and [69].

Rudolf et al. [70] compared $\mathrm{WCCoCr}, \mathrm{Cr}_{3} \mathrm{C}_{2}+25 \mathrm{NiC}$, WC+CrC+Ni coatings with and without impregnation deposited on $13 \mathrm{Cr} 4 \mathrm{Ni}$ steel with uncoated steel substrate using at two cavitation rigs: cavitating orifice and cavitating jet. The results showed that the tested coatings have very low CER. CER of the coatings were lower than that of uncoated steel.

The cited examples and other works [71-73] indicate that an important aspect in the case of coatings is not only the mechanical properties of the substrate or the coating itself, but also 
the properties of the whole coating-substrate system, including structural properties [69]. Particular attention should also be paid to the coating application parameters and the application method.

\section{SUMMARY}

In the paper correlations of selected mechanical properties (hardness, yield strength, tensile strength, strain energy and fatigue strength) with resistance to cavitation erosion have been presented. The influence of grain size on cavitation resistance of material and the possibilities of increasing cavitation erosion resistance is also shown. The exponential correlation between hardness, ultimate tensile stress, yield strength and CER is shown. However, with increasing hardness increases brittleness and brittle materials have low CER. The other property, which highly affects CER, is fatigue strength. The relationship between fatigue strength and mean depth penetration as well as maximum erosion rate has been proved several times. Fatigue approach has been used for predicting mass or volume loss of bulk steels exposed to cavitation.

Besides mechanical and endurance properties, structural properties, especially the grain size and the type of steel (austenitic, martensitic, ferritic), are also important for $C E R$. The smaller the grain size, the higher the CER. Modification of surface layer by applying coatings or heat treatment allows increasing CER.

Deposition of hard coatings increase CER in most cases. However, the correlation between coating properties and CER of the coating-substrate system is not simple. The mechanical properties of the coatings depend on deposition parameters. Besides hardness, adhesion is the other key property highly affects CER. In case of HVOF coatings, the grain size of the powder used for the coating is also an equally important aspect for CER.

\section{REFERENCES}

1. Chahine G. L., Franc J.-P., Karimi A.: Advanced Experimental and Numerical for Cavitation Erosion Prediction. Kim K., Chahine G. L., Franc J.-P., Karimi A. [eds], Springer International Publishing, Dordrecht, 2014.

2. Krella A. K.: Influence of cavitation intensity on X6CrNiTi18-10 stainless steel, Wear 258 (2005) 604-613.

3. Heymann F. J.: Erosion by liquids - The mysterious murderer of metals, Mach. Des. 1970.

4. Kumar P., Saini R. P.: Study of cavitation in hydro turbines - A review, Renewable and Sustainable Energy Reviews 14 (2010) 374-383.

5 . Brennen Ch.E.: Cavitation and bubble Dynamics. Oxford University Press, New York, 1977.

6. Hubballi B. V., Sondur V. B.: A Review on the prediction on cavitation erosion inception in hydraulic control valves, IJETAE 3.1 (2013) 110-119.

7. Ozonek J.: Application of Hydrodynamic Cavitation in Environmental Engineering. Taylor \& Francis Group, London, 2012.

8. Gogate P. R., Kabadi A. M.: A review of applications of cavitation in biochemical engineering/biotechnology, Biochemical Engineering Journal 44 (2009) 60-72. 
9. Karimi A., Martin J. L.: Cavitation erosion of materials. International Metals Reviews 31 (1986) $1-26$.

10 . Espitia L. A., Dong H., Li X. Y., Pinedo C. E., Tschiptschin A. P.: Cavitation erosion resistance and wear mechanisms of active Green low temperature plasma nitrided AISI 410 martensitic stainless steel, 332-333 (2015) 1070-1079.

11. Jasionowski R., Zasada D., Grabin J.: Mechanizm niszczenia stopów intermetalicznych poddanych erozji kawitacyjnej, Zeszyty Naukowe Akademii Morskiej w Szczecinie, 5 (2005) 257-266.

12. Feller H. G., Kharrazi Y.: Cavitation erosion of metals and alloys, Wear 93 (1984) 249-260.

13. Thiruvengadam A., Waring S. J.: Mechanical properties of metals and their cavitation damage resistance, Journal of Ship Res 10 (1966) 1-9.

14. Lin C., Zhao Q., Zhao X., Yang Y.: Cavitation erosion of metallic materials. IJGE 4 (2018) 1-8.

15. Richman R. H., McNaughton W. P.: Correlation of cavitation properties of metals erosion behavior with mechanical properties of metals. Wear 140 (1990) 63-82.

16. Chang S. C., Weng W. H., Chen H. C., Lin S. J., Chung P. C. K.: The cavitation erosion of FeMn-Al. Alloys. Wear (181-183 (1995) 511-515.

17 . Kwok C. T., Man H. C., Cheng F. T.: Cavitation erosion and damage mechanisms of alloys with duplex structures. Materials Science and Engineering A242 (1998) 108-120.

18 . Tzanakis I., Bolzoni L., Eskin D. G., Hadfield M.: Evaluation of cavitation erosion behavior of commercial steel grades used in the design of fluid machinery. Metallurgical and Materials Transactions A: Physical Metallurgy and Materials Science 48 (2017) 2193-2206.

19 . Hattori S., Ishikura R., Zhang Q.: Construction of database on cavitation erosion and analyses of carbon steel data. Wear 257 (2004) 1022-1029.

20 . Hattori S., Ishikura R.: Revision of cavitation erosion database and analysis of stainless steel data. Wear 268 (2010) 109-116.

21. Howard, R.L., Ball, A.: Mechanisms of cavitation erosion of TiAl -based titanium alumine intermetalic alloys. Acta Metallurgica 44 (1996) 3157-3168

22. Hattori S., Kitagawa T.: Analysis of cavitation erosion resistance of cast iron and nonferrous metals based on database and comparison with carbon steel data. Wear 269 (2010) 443-448

23. Szkodo M.: Erozja kawitacyjna materiałów konstrukcyjnych, Gdańsk, 2008.

24. Garcia R., Hammit F. G.: Cavitation damage and correlations with material and fluid properties. Journal of Basic Enineering 89 (1967) 753-763.

25 . Bregliozzi G., Di Schino A., Ahmed S. I. U., Kenny J. M., Haefke H.: Cavitation wear behaviour of austenitic stainless steel with different grain sizes. Wear 258 (2005) 503-510.

26. Chauhan A. K., Goel D. B., Prakash S.: Erosion behavior of hydroturbine steel. Bulletin of Materials Science 31 (2008) 115-120.

27. Niederhofer P., Huth S.: Cavitation erosion resistance of high interstinal CrMnCN austenitic stainless steel. Wear 301 (2013) 457-466.

28 . Thapliyal S., Dwivedi D. K.: On cavitation erosion behavior of friction processed surface of cast nickel aluminium bronze. Wear 376-377 (2017) 1030-1042.

29 . Wantang F., Yangzeng Z., Xiaokui H.: Resistance of a high nitrogen austenitic steel to cavitation erosion. Wear 249 (2001) 788-791. 
30. Sreedhar B.K., Albert S. K., Pandit A.B.: Cavitation damage and measurements - A review. Wear 372-373 (2017) 177-196.

31. Peng K., Kang C., Li G., Matsuda K., Soyama H.: Effect of heat treatment on the cavitation erosion resistance of stainless steel. Materials and Corrosion (2017) 1-9.

32. Nair R. B., Arora H.S., Mukherjee S., Singh S., Grewal H. S.: Exceptionally high cavitation erosion and corrosion resistance of a high entropy alloy. Ultrasonics Sonochemistry 41 (2018) 252-260.

33. Mann B.S.: Boronizing of cast martensitic chromium nickel stainless steel and its abrasion and cavitation-erosion behaviour. Wear 208 (1997) 125-131.

34 . Singh R., Tiwari S. K., Mishra S. K.: Cavitation erosion In hydraulic turbine components and mitigation by coatings: current status and future needs. Materials Engineering and Performance 21 (2012) 1539-1551.

35 . Będkowski W., Gasiak G., Lachowicz C., Lichtarowicz A., Lagoda T., Macha E.: Relations between cavitation erosion resistance of materials and their fatigue strength under random loading. Wear 230 (1999) 201-209.

36 . Knapp R. T., Daily J. W., Hammit F.G.: Cavitation, McGraw-Hill, New York 1970.

37. Richman R. H., McNaughton W. P.: A metallurgical approach to improved cavitation-erosion resistance. Materials Engineering And Performance 6 (1997) 633-641.

38 . Chen W., Gu C., Zhao K., Shen F.: Correlation of cavitation erosion resistance and mechanical properties of some engineering steels. Materials Science 41 (2006) 2151-2153.

39 . Hattori S., Takamoto I.: Cavitation erosion resistance of plastics. Wear 271 (2011) 1103-1108.

40 . Fortes Patella R., Choffat T., Jean-Luc Reboud J-L, Archer A.: Mass loss simulation in cavitation erosion: Fatigue criterion approach. Wear 300 (2013) 205-215.

41 . Krella A.K., Krupa A.: Effect of cavitation intensity on degradation of X6CrNiTi18-10 stainless steel. Wear 408-409 (2018) 180-189.

42. Di Schino A., Barteri M., Kenny J. M.: Effects of grain size on the properties of a low nickel austenitic stainless steel. Journal of Materials Science 38 (2003) 4725-4733.

43. Wang Z., Zhang X., Cheng J., Lin J., Zhou Z.: Cavitation erosion resistance of Fe-based amorphous/nanocrystal coatings prepared by high-velocity arc spraying. Journal of Thermal Spray Technology 23 (2014) 742-749.

44. Hajian M., Abdollah-Zadeh A., Razaei-Nejad S. S., Assadi H., Hadavi S. M. M., Chung K., Shokouhimehr M.: Improvement in cavitation erosion resistance of AISI $316 \mathrm{~L}$ stainless steel by friction stir processing. Applied Surface Science 308 (2014) 184-192.

45 . Liu W., Zheng Y. G., Liu C. S., Yao Z. M., Ke W.: Cavitation erosion behaviour of Cr-n-N stainless steel in comparison with 0Cr13Ni5Mo stainless steel. Wear 254 (2003) 713-722.

46. Lin J., Wang Z., Cheng J., Kang M. Fu X., Hong S.: Effect of initial surface roughness on cavitation erosion resistance of arc-spayed Fe-based amorphous/nanocrystalline coating. Coatings 7 (2017) 200-209.

47 . Xiaojun Z., Procopiak L.A.J., Souza N. C., d'Oliveira A.S.C.M.: Phase transformation during cavitation erosion of Co stainless steel. Materials Science and Engineering A 358 (2003) 1992014.

48 . Li D. G., Chen D. R., Liang P.: Enhancement of cavitation erosion resistance of 316L stainless steel by adding molybdenum. Ultrasonics Sonochemistry 35 (2017) 375-381.

49 . Allenstein A. N., Lepienski C. M., Buschinelli A. J. A., Brunatto S. F.: Improvement of the cavitation erosion resistance for low-temperature plasma nitrided Ca-6NM martensitic stainless 
steel. Wear 309 (2014) 159-165.

50 . Verma S., Dubey P., Selokar A. W., Dwivedi D. K., Chandra R.: Cavitation erosion behaviour of nitrogen ion implanted 13Cr4Ni steel. Transaction of the Indian Institute of Metals 70 (2017) 957-965.

51. Lamana M. S. Pukasiewicz A. G. M., Sampath S.: Influence of kobalt kontent and HVOF deposition process on the cavitation erosion resistance of WC-Co coatings. Wear 398-399 (2017) 209-219.

52 . Lavigne S., Pougoum F., Savoie S., Martinu L., Klemberg-Sapiecha E., Schulz R.: Cavitation erosion behaviour of HVOF CaviTec Coatings. Wear 386-387 (2017) 90-98.

53 . Kumar A., Sharma A., Goel S. K.: Erosion behaviour of WC-10Co-4Cr on 23-8-N nitronic steel by HVOF thermal spraying. Applied Surface Science 370 (2016) 418-426.

54 . Momeni S., Tillmann W., Pohl M.: Composite cavitation resistant PVD coatings based on NiTi thin films. Materials and Design 110 (2016) 830-838.

55 . Krella A.: An experimental parameter of cavitation erosion resistance for TiN coatings. Wear 270 (2011) 252-257.

56. Krella A.: Cavitation erosion of TiN and $\mathrm{CrN}$ coatings deposited on different substrates. Wear 297 (2013) 992-997.

57 . Stella J., Schüller E., Heßing C., Hamed O. A., Pohl M., Stöver D.: Cavitation erosion of plasmasprayed NiTi coating. Wear 260 (2006) 1020-1027.

58. Wang Y., Liu J., Kang N., Darut G., Poirier T., Stella J., Liao H., Planche M. P.: Cavitation erosion of plasma-sprayed CoMoCrSi coatings. Tribology International 102 (2016) 429-435.

59 . Godoy C., Mancosu R. D., Lima M. M., Brandao D., Housden J., Avelar-Batista J. C.: Influence of plasma nitriding and PAPVD $\mathrm{Cr}_{1-\mathrm{x}} \mathrm{N}_{\mathrm{x}}$ coating on the cavitation erosion resistance of an AISI 1045 steel. Surface Coatings Technology 200 (2006) 5370-5378.

60 . Kim J., Lee M.: A study on cavitation erosion and corrosion behaviour of Al-, $\mathrm{Zn}$-, $\mathrm{Cu}-$, and $\mathrm{Fe}-$ based coatings prepared by arc spraying. Journal of Thermal Spray Technology 19 (2010) 12241230 .

61 . Lo K. H., Cheng F. T., Kwok C- T., Man H. C.: Improvement of cavitation erosion resistance of AISI 316 stainless steel by laser surface alloying using fine WC powder. Surface and Coatings Technology 165 (2003) 258-267.

62 . Wu Y., Hong S., Zhang J., He Z., Guo W., Wang Q., Li G.: Microstructure and cavitation erosion behaviour of WC-Co-Cr coating $1 \mathrm{Cr} 18 \mathrm{Ni}$ Ti stainless steel by HVOF thermal starying. International Journal of Refractory Metals and Hard Materials 32 (2012) 21-26.

63 . Taillon G., Pougoum F., Lavigne S., Ton-That L., Schulz R., Bousser E., Savoie S., Martinu L., Klemberg-Sapiecha E.: Cavitation erosion mechanisms in stainless steel and in composite metalceramic HVOF coating. Wear 364-365 (2016) 201-210.

64. Wang Y., Stella J., Darut G., Poirier T., Liao H., Planche M. P.: APS prepared NiCrBSi-YSZ composite coatings for protection against cavitation erosion. Journal of Alloys and Compounds 699 (2017) 1095-1103.

65 . Zhang H., Gong Y., Chen X., McDonald A., Li H.: A comparative study of cavitation erosion resistance of several HVOF-sprayed coatings in deionized water and artificial seawater. Journal of Thermal Spray Technology 28 (2019) 1060-1071.

66. Hou G., Ren Y., Zhang X., Dong F., An Y., Zhao X., Zhou H., Chen J.: Cavitation erosion mechanisms in Co-based coatings exposed to seawater. Ultrasonics -Sonochemistry 60 (2020) 1047-59. 
67. Szala M., Walczak M., Pasierbiewicz K., Kamiński M.: Cavitation erosion and sliding wear mechanisms of AlTiN and TiAlN films deposited on stainless steel substrate. Coatings 9340 (2019).

68 . Deng W., Zhao X., Ren Y., Hao E., Han J., An Y., Zhou H., Chen J.: Influence of epoxy resin on the microstructure and cavitation erosion of as-sprayed 8YSZ coating. Ceramics International 45 (2019) 5693-5702.

69 . Krella A.K.: An approach to evaluate the resistance of hard coatings to shock loading. Surface \& Coatings Technology 205 (2010) 2687-2695.

70 . Rudolf P., Julis M., Klakurkova L., Gejdos P., Hudec M.: Cavitation erosion testing of different cavitation-resistant materials and coatings using the cavitating jet method. IOP Conf. Series: Earth and Enviromental Science 240 (2019) 57-62.

71. Ding H., Jiang S., Xu J.: Cavitation erosion resistance of $\mathrm{ZrC}$ nanoceramic coating. Journal of Engineering Tribology (2019) 1-9.

72 . Qiao L., Wu Y., Hong S., Zhang J., Shi W., Zheng Y.: Relationships between spray parameters, microstructure and ultrasonic cavitation erosion behavior of HVOF sprayed Fe-based amphous/nanocrystalline coating. Ultrasonics - Sonochemistry 39 (2017) 39-46.

73 . Matikainen V., Peregrina S. R., Ojala N., Koivuluoto H., Schubert J., Houdkova S., Vuoristo P.: Erosion wear performance of $\mathrm{WC}-10 \mathrm{Co} 4 \mathrm{Cr}$ and $\mathrm{Cr} 3 \mathrm{C} 2-25 \mathrm{NiCr}$ coatings sprayed with highvelocity thermal spray processes. Surface \& Coatings 370 (2019) 196-212. 